\title{
Concurrent colonic mucosa-associated lymphoid tissue lymphoma and adenoma diagnosed after a positive fecal occult blood test: a case report
}

\author{
Pei-Chiang Lin ${ }^{1}$, Jinn-Shiun Chen ${ }^{2}$, Po Deng ${ }^{3}$, Chih-Wei Wang ${ }^{4}$, Chiung-Huei Huang ${ }^{5}$, Reiping Tang ${ }^{2,6}$, \\ Jy-Ming Chiang ${ }^{2}$, Chien-Yuh Yeh², Pao-Shiu Hsieh², Wen-Sy Tsai ${ }^{2}$ and Sum-Fu Chiang ${ }^{2^{*}}$
}

\begin{abstract}
Background: Colonic lymphoma is an uncommon presentation of extranodal lymphoma. Colonic mucosaassociated lymphoid tissue lymphoma is a different entity from gastric mucosa-associated lymphoid tissue lymphoma, and very rare. The presentation and management of colonic mucosa-associated lymphoid tissue are highly variable in the literature.

Case presentation: We report the case of a 59-year-old Taiwanese man who underwent a colonoscopy after a positive test for fecal occult blood. His past history included hypertension and hyperthyroidism. The colonoscopy revealed an adenomatous polyp and mucosa-associated lymphoid tissue lymphoma. We successfully performed a polypectomy and endoscopic mucosal resection. The lymphoma was staged according to the Ann Arbor system modified by Musshoff as E-I. Our patient showed no lymphoma recurrence over a 3-year follow-up.

Conclusions: Endoscopic mucosal resection for colonic mucosa-associated lymphoid tissue lymphoma without disseminated disease may be feasible. We successfully used colonoscopic treatment without adjuvant therapy to treat early-stage pathogen-free colonic mucosa-associated lymphoid tissue lymphoma.
\end{abstract}

Keywords: Colon lymphoma, Endoscopic mucosal resection, Mucosa-associated lymphoid tissue lymphoma

\section{Background}

The gastrointestinal tract is the most common site for extranodal lymphomas, with the colon being the least involved area $[1,2]$. Colon lymphomas account for $15-20 \%$ of gastrointestinal lymphomas [1], $1.4 \%$ of all nonHodgkin's lymphomas [3], and $1 \%$ of all colorectal malignancies [4]. Mucosa-associated lymphoid tissue (MALT) lymphoma represents the third most common nonHodgkin's lymphoma [5], and one of the two most common types of gastrointestinal non-Hodgkin's lymphoma [2]. Unlike gastric MALT lymphoma, colonic MALT lymphoma is very rare. The presentation of colonic MALT lymphoma is highly variable, and its management is not

\footnotetext{
* Correspondence: sumfuchiang@gmail.com

${ }^{2}$ Division of Colon and Rectal Surgery, Chang Gung Memorial Hospital, 5,

Fu-Hsing Street, Kuei-Shan Hsiang, Tao-Yuan, Linkou, Taiwan

Full list of author information is available at the end of the article
}

clear [6-11]. We treated a male patient with asymptomatic colonic MALT lymphoma, which was successfully resected by colonoscopy.

\section{Case presentation}

Our patient was a 59-year-old Taiwanese, well-nourished man with a history of hypertension for 4 years and hyperthyroidism for one more year that were well controlled. He was a heavy smoker (1-2 packs per day) for 30 years, and had only quit for a few months. He had received a lumbar laminectomy to treat spinal stenosis 6 years previously. He had no relevant family history. He had developed thyrotoxic exophthalmos 2-3 months prior to presentation and received steroid pulse therapy for compressive optic neuropathy. The symptoms were relieved for 2-3 months after the steroid therapy. 
He visited our clinic after a positive fecal occult blood test $(132 \mathrm{ng} / \mathrm{mL})$ but reported no change to his bowel habits. A physical examination revealed bilateral eyes proptosis and mild obesity. Complete colonoscopy revealed one flat polyp, $0.3 \mathrm{~cm}$ in size, located $35 \mathrm{~cm}$ from his anal verge, and another polypoid polyp with a wide base, $2.0 \mathrm{~cm}$ in size with a slightly irregular border, located $25 \mathrm{~cm}$ from his anal verge. The smaller polyp was removed by polypectomy. We biopsied the larger polyp because of its wide base. A pathological examination of the specimen revealed it was a hyperplastic polyp. We did not perform an endoscopic ultrasound because we intended to directly remove the polyp. The polyp was then removed by endoscopic mucosal resection followed by clipping (Fig. 1). There were no unexpected events after the procedure.

A pathological examination of the removed polyps identified the smaller polyp as adenomatous and the larger one as a MALT lymphoma, with a polypoid colonic mucosa and atypical lymphoid cells infiltrating the lamina propria (Fig. 2a). An immunohistochemical study demonstrated that the larger polyp was positive for CD20, CD5, and Bcl-2, and negative for CD10 and cyclin D1 (Fig. 2b). These results supported our diagnosis of extranodal marginal zone lymphoma of MALT type. However, when reviewed by a pathologist, the margins of the endoscopic mucosal resection specimen were found to be positive (cauterized margin with lymphoma cells; Fig. 2c), although it was free on gross examination.
Our patient was transferred to the hematologist's clinic, and further studies revealed no disease dissemination. A physical examination revealed no palpable lymph nodes, no petechiae, and no hepatosplenomegaly. A computed tomography scan revealed no metastatic tumors, nor enlarged lymph nodes. A bone marrow biopsy of his right iliac bone revealed small aggregates of small lymphoid cells but an immunohistochemical study did not suggest any MALT lymphoma involvement. An otorhinolaryngologist found no abnormalities in his ear drums, nose, oral cavity, nasopharynx, or vocal cord. The tumor stage was E-I according to the Ann Arbor staging system modified by Musshoff [12].

Our patient had regular follow-up appointments with the hematologist and proctologist without any signs of lymphoma recurrence for 3 years. Two years after his initial presentation, a colonoscopy revealed a transverse colon adenoma and a rectal hyperplastic polyp. The previous site of the MALT lymphoma was free of tumor, and no other evidence of MALT lymphomas was found. A polypectomy was performed with no unexpected events.

According to his history and throughout his clinical course, our patient had no symptoms or signs of a peptic ulcer. We did not perform a panendoscopy nor test for Helicobacter pylori. There were no other symptoms or signs compatible with autoimmune diseases or viral infection. We did not test for Epstein-Barr virus (EBV). During the last 2 years, he has had no epigastralgia, no acid reflux, and no tarry stool.
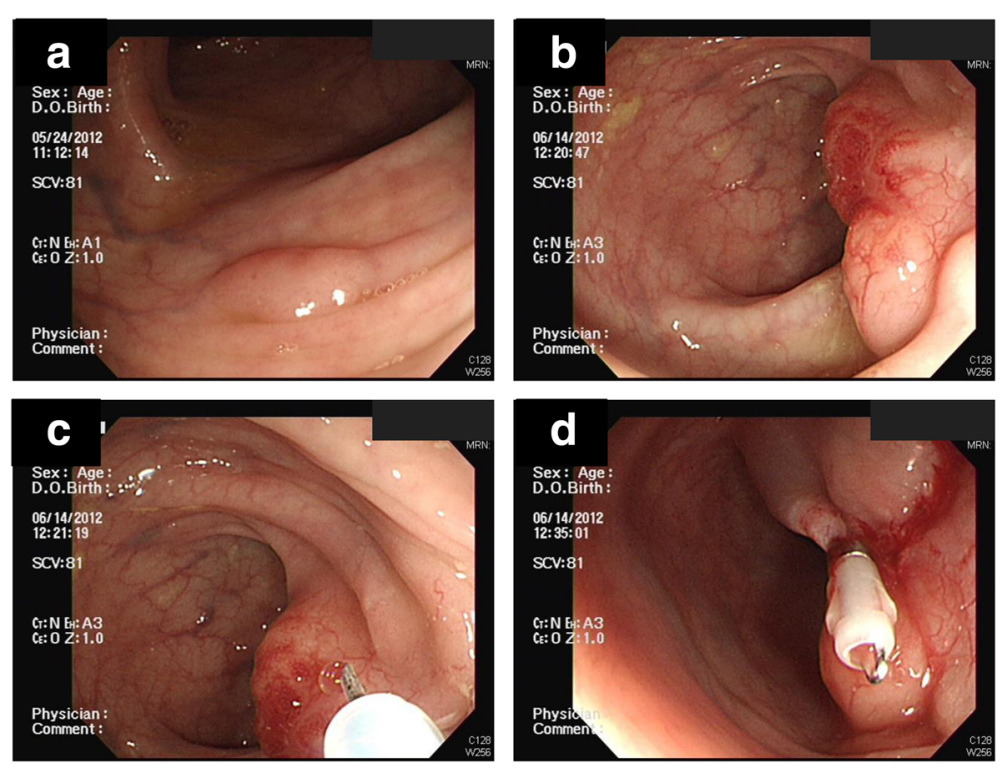

Fig. 1 Complete colonoscopy revealed a flat polyp located $35 \mathrm{~cm}$ from the anal verge (a). Pathology identified it as an adenomatous polyp. Another $2.0 \mathrm{~cm}$ polypoid polyp was found $25 \mathrm{~cm}$ from the anal verge (b). It was polypoid with a wide base, slightly irregular border, and an irregular vascular pattern with mild inflammatory changes. We removed the second lesion (shown in b) by endoscopic mucosal resection (c), followed by the application of one hemoclip for wound closure (d) 


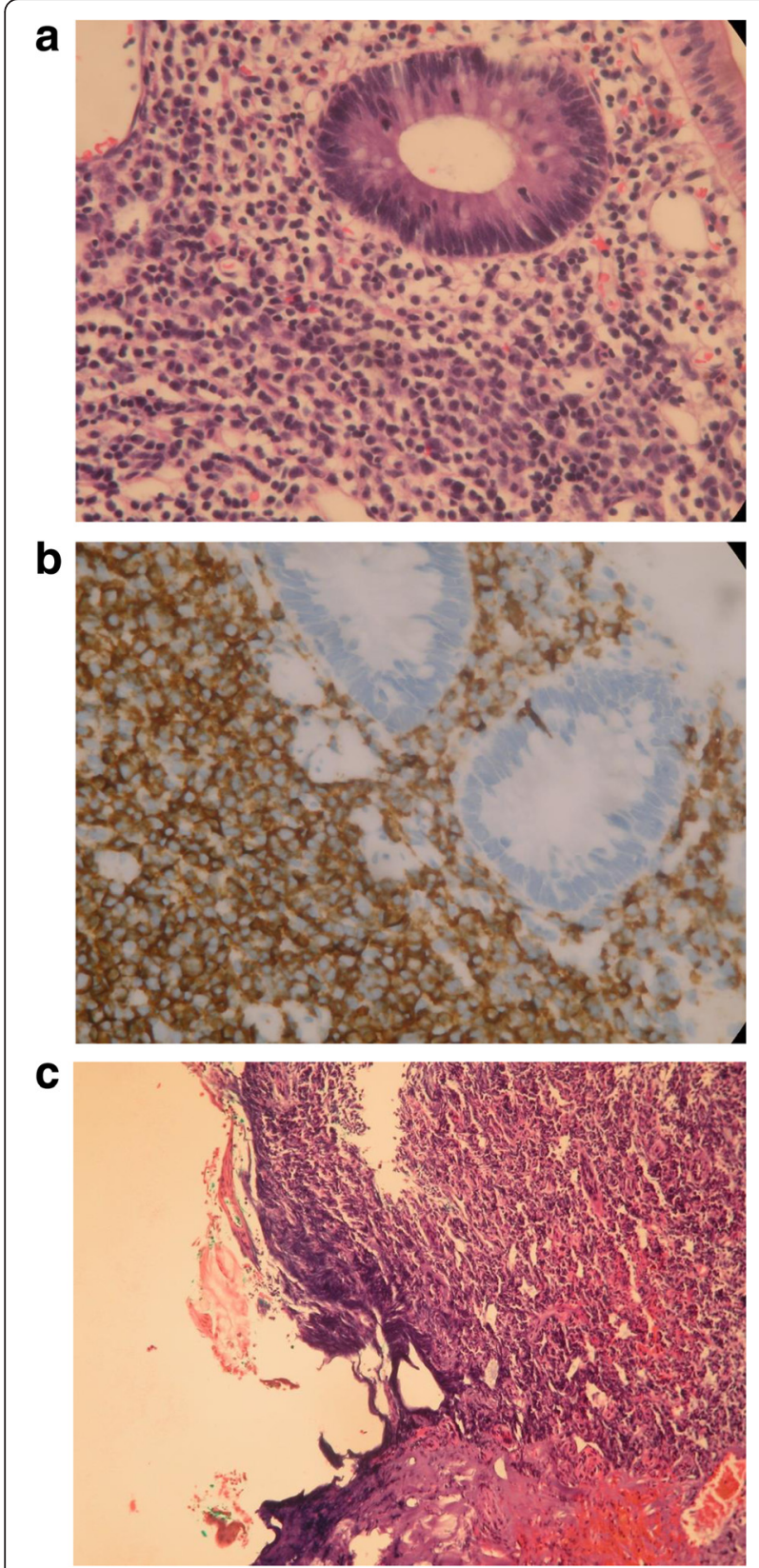

Fig. 2 Pathology of the larger polypoid polyp revealed polypoid colonic mucosa with atypical lymphoid cells infiltrating the lamina propria (a). An immunohistochemical study found that the specimen was positive for CD20, CD5, and BCl-2, and negative for CD10 and cyclin D1, which supported the diagnosis of extranodal marginal zone lymphoma of mucosa-associated lymphoid tissue lymphoma type (b). The resected specimen is positive for lymphoma cells, which are present at the cauterized margin (left side of the figure; hematoxylin and eosin stain, 100x) (c)

\section{Discussion}

MALT-type lymphoma was first defined by Isaacson and Wright in 1983 [13]. Colon lymphoma is a rare example of extranodal lymphoma. Colonic MALT lymphoma is much rarer than gastric MALT lymphoma, which is related to $H$. pylori infection [1, 2, 9]. MALT development is thought to be related to chronic antigen stimulation [7]. It comprises morphologically heterogeneous small B-cells, and typically infiltrates the epithelium [14]. Colon MALT lymphoma might be a more "benign" disease given that most cases reported in the literature were stage E-I and EII diseases without dissemination [6-11].

The most well-known gastrointestinal lymphoma is gastric MALT lymphoma. Gastric MALT lymphoma has a strong predisposing factor, $H$. pylori infection. The prevalence of $H$. pylori infection is as high as $80 \%$ in patients with gastric MALT lymphoma $[15,16]$. However, not all patients are responsive to $H$. pylori eradication $[17,18]$. Furthermore, the recurrence of gastric MALT lymphoma is accompanied by $H$. pylori infection in only $0-57 \%$ of patients $[17,19]$. Asano et al. reviewed $H$. pylori-negative gastric MALT lymphomas and suggested that possible mechanisms include antibiotic eradication for non- $H$. pylori bacteria and the immunomodulatory effect of clarithromycin [20].

Other associated diseases, such as EBV infection and autoimmune diseases, have also been reported in the literature [21]. Kaneko et al. reported the resemblance between tonsil MALT lymphoma and EBV-related tonsillar hyperplasia [21]. These two diseases can only be differentiated by immunohistochemical studies. MALT lymphomas of sites other than the stomach have also been reported. They include lung [22], tonsil [21], and ocular adnexa [23]. Most of them these lymphomas had a benign or premalignant nature with an excellent prognosis [22, 23].

Unlike gastric MALT lymphoma, colon MALT lymphoma does not have any obvious predisposing factors. Although chronic antigen stimulation is a hypothetical etiology [7], it was not present in most reported colon MALT lymphomas. Several cases with MALT lymphoma received and responded to $H$. pylori eradication $[7,10]$. Our patient had a history of hyperthyroidism and exophthalmos, but associations between other diseases and colon MALT lymphoma had not been reported in the literature until 2-3 years ago. Jain et al. reported a MALT lymphoma of the cecum presenting as an acute bowel obstruction; however, no $H$. pylori nor other etiologies could be identified [24]. Terada reported the case of an 18-year-old man with a 2year history of ulcerative colitis who developed MALT lymphoma [25]. His diagnosis was made by colonoscopic biopsy, and he only received treatment for his ulcerative colitis after that [25].

The presentation and management of colonic MALT lymphoma are still highly variable. The symptoms include a positive test for fecal occult blood, constipation, abdominal pain, gastro-intestinal bleeding, and tenesmus, or it may be asymptomatic [6-11]. Management includes local resection, colectomy, $H$. pylori eradication, chemotherapy, and radiotherapy $[6-8,10,11]$. To the 
best of our knowledge, our patient's case is the first report of successful management by colonoscopy for MALT lymphoma. No antibiotic eradication was applied. Our patient has one of the longest follow-up times without recurrence (3 years) reported in the literature [6]. However, the rarity of colon MALT lymphomas means that more cases are required for comparison.

\section{Conclusion}

Although more and more MALT lymphomas have been reported, colon involvement is still relatively rare. We report the case of a patient with a colonic MALT lymphoma with previous hyperthyroidism. Our patient was asymptomatic and underwent endoscopic mucosal resection without antibiotic eradication for a stage E-I tumor. $\mathrm{He}$ attended regular follow-up appointments without lymphoma recurrence for 3 years. A "wait and see" policy for resectable stage E-I colonic MALT lymphoma without pathogens seems to be a reasonable course of action. However, more studies about standard management for the disease are needed.

\section{Consent}

Written informed consent was obtained from the patient for publication of this case report and any accompanying images. A copy of the written consent is available for review by the Editor-in-Chief of this journal.

\section{Competing interests}

The authors declare that they have no competing interests.

\section{Authors' contributions}

PCL and SFC carried out the endoscopic excision. JSC, PD, CHH, CYY, PSH, WST, and SFC contributed to the discussion of this case. CWW performed the pathologic analysis. PCL, CHH, RT, JMC, and SFC participated in its design and coordination and helped to draft the manuscript. All authors read and approved the final manuscript.

\section{Acknowledgements}

We would like to thank the consultants of the Division of Hematology-Oncology, and the Division of Endocrinology and Metabolism, Department of Internal Medicine, Chang Gung Memorial Hospital. We also thank the outstanding team of surgeons and endoscopic therapists of the Division of Colon and Rectal Surgery, Chang Gung Memorial Hospital.

\section{Author details \\ ${ }^{1}$ Division of Colon and Rectal Surgery, Lin Shin Hospital, Taichung, Taiwan. ${ }^{2}$ Division of Colon and Rectal Surgery, Chang Gung Memorial Hospital, 5, Fu-Hsing Street, Kuei-Shan Hsiang, Tao-Yuan, Linkou, Taiwan. ${ }^{3}$ Division of Hematology-Oncology, Department of Internal Medicine, Chang Gung Memorial Hospital, Linkou, Taiwan. ${ }^{4}$ Department of Pathology, Chang Gung Memorial Hospital, Linkou, Taiwan. ${ }^{5}$ Division of Endocrinology and Metabolism, Department of Internal Medicine, Chang Gung Memorial Hospital, Linkou, Taiwan. ${ }^{6}$ Chang Gung University College of Medicine, Linkou, Taiwan.}

Received: 18 June 2015 Accepted: 14 January 2016

Published online: 27 January 2016

\section{References}

1. Koch P, del Valle F, Berdel WE, Willich NA, Reers B, Hiddemann W, et al. Primary gastrointestinal non-Hodgkin's lymphoma: I. Anatomic and histologic distribution, clinical features, and survival data of 371 patients registered in the German Multicenter Study GIT NHL 01/92. J Clin Oncol. 2001;19(18):3861-73.

2. Psyrri A, Papageorgiou S, Economopoulos T. Primary extranodal lymphomas of stomach: clinical presentation, diagnostic pitfalls and management. Ann Oncol. 2008;19(12):1992-9.

3. Doolabh N, Anthony T, Simmang C, Bieligk S, Lee E, Huber P, et al. Primary colonic lymphoma. J Surg Oncol. 2000;74(4):257-62.

4. Fan CW, Changchien CR, Wang JY, Chen JS, Hsu KC, Tang R, et al. Primary colorectal lymphoma. Dis Colon Rectum. 2000;43(9):1277-82.

5. Pileri SA, Milani M, Fraternali-Orcioni G, Sabattini E. From the REAL-classification to the upcoming WHO-scheme: a step towards universal categorization of lymphoma's entities? Ann Oncol. 1998;9(6):607-12.

6. Schmid C, Vazquez JJ, Diss TC, Isaacson PG. Primary B-cell mucosaassociated lymphoid tissue lymphoma presenting as a solitary colorectal polyp. Histopathology. 1994;24:357-62

7. Raderer M, Pfeffel F, Pohl G, Mannhalter C, Valencak J, Chott A. Regression of colonic low grade B cell lymphoma of the mucosa associated lymphoid tissue type after eradication of Helicobacter pylori. Gut. 2000;46:133-5.

8. Lee YG, Lee S, Han SW, Lee JS. A case of multiple mucosa-associated lymphoid tissue (MALT) lymphoma of the colon identified as simple mucosal discoloration. J Korean Med Sci. 2005;20(2):325-8.

9. Kanneganti K, Bhavna B. Mucosa-associated lymphoid tissue lymphoma presenting as massive gastrointestinal bleeding: a case report. Case Rep Gastroentrol. 2008;2:296-300.

10. Ferreira R, Torres J, Raposo J, Duarte MB, Pereira MJ, Carvalheiro J, et al. Multifocal colonic mucosa-associated lymphoid tissue lymphoma with synchronous tubular adenoma: a coincidental association? Int J Colorectal Dis. 2011;26(9):1221-2.

11. Akasaka R, Chiba T, Dutta AK, Toya Y, Mizutani T, Shozushima T, et al. Colonic mucosa-associated lymphoid tissue lymphoma. Case Rep Gastroenterol. 2012;6(2):569-75.

12. Musshoff K. Clinical staging classification of non-Hodgkin's lymphomas (author's transl). Strahlentherapie. 1977;153(5):218-21.

13. Isaacson PG, Wright DH. Malignant lymphoma of mucosa-associated lymphoid tissue. A distinctive type of B-cell lymphoma. Cancer. 1983;52:1410-6.

14. Isaacson PG. Update on MALT lymphomas. Best Pract Res Clin Haematol. 2005:18:57-68.

15. Asenjo LM, Gisbert JP. Prevalence of Helicobacter pylori infection in gastric MALT Iymphoma: a systematic review. Rev Esp Enferm Dig. 2007;99:398-404.

16. Nakamura S, Yao T, Aoyagi K, lida M, Fujishima M, Tsuneyoshi M. Helicobacter pylori and primary gastric lymphoma. A histopathologic and immunohistochemical analysis of 237 patients. Cancer. 1997;79:3-11.

17. Gisbert JP, Calvet X. Review article: common misconceptions in the management of Helicobacter pylori-associated gastric MALT-lymphoma. Aliment Pharmacol Ther. 2011;34(9):1047-62.

18. Chen LT, Lin JT, Tai JJ, Chen GH, Yeh HZ, Yang SS, et al. Long-term results of anti-Helicobacter pylori therapy in early-stage gastric high-grade transformed MALT lymphoma. J Natl Cancer Inst. 2005;97:1345-53.

19. Hong SS, Jung HY, Choi KD, Song HJ, Lee GH, Oh TH, et al. A prospective analysis of low-grade gastric malt lymphoma after Helicobacter pylori eradication. Helicobacter. 2006;11(6):569-73.

20. Asano N, lijima K, Koike T, Imatani A, Shimosegawa T. Helicobacter pylorinegative gastric mucosa-associated lymphoid tissue lymphomas: a review. World J Gastroenterol. 2015;21(26):8014-20.

21. Kaneko Y, Kojima M, Suzuki S, Takada A, Yamagishi H, Nakazato Y, et al. Atypical interfollicular hyperplasia of tonsils resembling mucosa-associated lymphoid tissue lymphoma: a clinicopathological, immunohistochemical study and Epstein-Barr virus findings in 12 cases. J Clin Exp Hematop. 2014;54(2):111-6.

22. Rubenstein JN, Beatty C, Kinkade Z, Bryan C, Hogg JP, Gibson LF et al. Extranodal marginal zone lymphoma of the lung: evolution from an underlying reactive lymphoproliferative disorder. J Clin Exp Pathol. 2015;5(1)

23. Kiesewetter B, Lukas J, Kuchar A, Mayerhoefer ME, Streubel B, Lagler H, et al. Clinical features, treatment and outcome of mucosa-associated lymphoid tissue (MALT) lymphoma of the ocular adnexa: single center experience of 60 patients. PLoS One. 2014;9(7):e104004.

24. Jain V, Misra S, Ahmad F, Rahul K, Singh A. MALT lymphoma of caecum presenting as acute intestinal obstruction: a case report. Indian J Surg. 2013; 75 Suppl 1:286-9.

25. Terada T. Extranodal marginal zone B-cell lymphoma of mucosa-associated lymphoid tissue (MALT lymphoma) in ulcerative colitis. Saudi J Gastroenterol. 2014;20(5):319-22. 